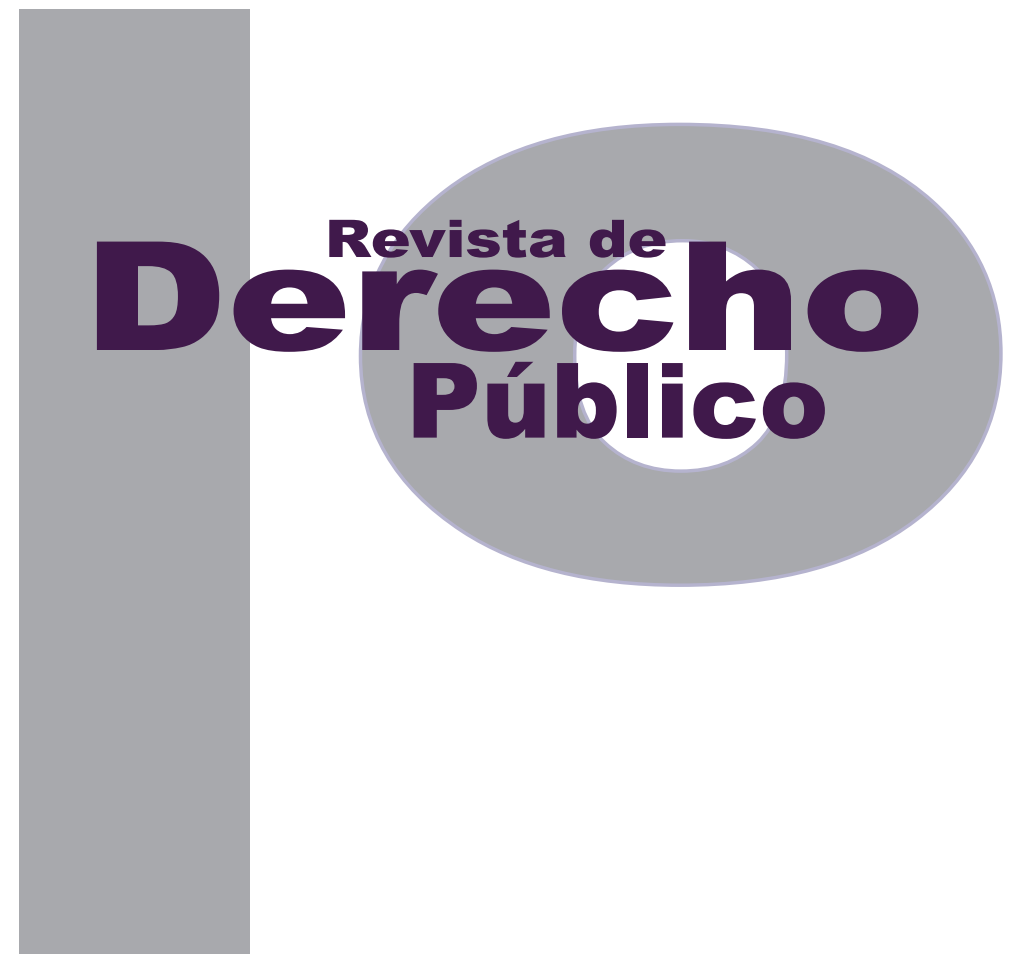

\title{
LA POLÍTICA DE MEJORA REGULATORIA: OPORTUNIDADES Y DESAFÍOS PARA COLOMBIA
}

\author{
INDIRA LATORRE \\ Artículo de reflexión \\ DOI: http://dx.doi.org/10.15425/redepub.33.2014.25 \\ Universidad de los Andes \\ Facultad de Derecho \\ Revista de Derecho Público N. ${ }^{\circ} 33$ \\ Julio - Diciembre de 2014. ISSN 1909-7778
}




\section{La política de mejora regulatoria: oportunidades y desafíos para Colombia}

\section{Resumen}

Actualmente, Colombia está negociando su ingreso a la Organización para la Cooperación y el Desarrollo Económicos (OCDE). Para obtener dicho ingreso, deberá, entre otras tareas, diseñar una política de mejora regulatoria. Este artículo busca introducir los ejes principales de esta política, con el fin de señalar las oportunidades y los desafíos de su implementación futura. La mayor oportunidad será su contribución al Estado constitucional y democrático de derecho, pero para sacar provecho de esto, primero se deberán afrontar algunos desafíos. El desafío principal será formular esta política teniendo en cuenta una noción amplia de regulación (Prosser, 2010).

Palabras clave: política de mejora regulatoria, Estado regulador, Estado constitucional.

\section{Better regulatory policy: opportunities and challenges for Colombia}

\section{Abstract}

Currently, Colombia is negotiating its membership to the OECD. To achieve its accession, Colombia shall, among other things, adopt a regulatory policy. This article aims to introduce the main axes of this policy in order to identify opportunities and challenges for its future implementation. The biggest opportunity will be its contribution to the constitutional and democratic state, but in order to take advantage of this, some challenges will need to be addressed first. The main challenge will be to formulate this policy taking into account a broad notion of regulation (Prosser, 2010).

Keywords: better regulatory policy, regulatory state, constitutional state.

\section{A política de melhoria regulatória: oportunidades e desafios para a Colômbia}

\section{Resumo}

Atualmente, a Colômbia está negociando sua entrada na Organização para a Cooperação e Desenvolvimento Econômico (OCDE). Para obter dita entrada, o país deverá, entre outras tarefas, desenhar uma política de melhoria regulatória. Este artigo busca introduzir os principais eixos desta política, com o fim de mostrar as oportunidades e os desafios de sua futura implementação. A maior oportunidade será sua contribuição ao Estado constitucional e democrático de direito, mas para tirar proveito disto, primeiro se deverão afrontar alguns desafios. O principal desafio será formular esta política levando em consideração uma noção ampla de regulação (Prosser, 2010).

Palavras-chave: política de melhoria regulatória, Estado regulador, Estado constitucional. 


\title{
La política de mejora regulatoria: oportunidades y desafíos para Colombia*
}

\author{
Indira Latorre**
}

\section{SUMARIO}

Introducción - I. LAS POLÍTICAS DE MEJORA REGULATORIA - A. Alcance y significado de la política de mejora regulatoria en la OCDE - B. La evolución de los lineamientos para el desarrollo de la política de mejora regulatoria - C. Principios para mejorar la calidad de la regulación - 1. Necesidad - 2. Proporcionalidad - 3. Transparencia - 4. Accesibilidad - 5. Simplicidad - 6. Accountability - II. UNA POLITICA DE MEJORA REGULATORIA PARA COLOMBIA - A. Las recomendaciones de la OCDE - B. Una hoja de ruta con desafíos y oportunidades - 1. Ampliar la visión sobre la regulación - 2. Promover la investigación jurídica sobre mejora regulatoria - 3. Emprender el camino hacia la democracia deliberativa - 4. Fortalecer la búsqueda de decisiones razonadas e imparciales - 5. Mejorar el diálogo entre poderes y sectores administrativos - 6. Revaluar la cultura jurídica y política - III. CONCLUSIONES - Referencias.

\footnotetext{
* Cómo citar este artículo: Latorre, I. (Diciembre, 2014). La política de mejora regulatoria: oportunidades y desafíos para Colombia. Revista de Derecho Público, 33. Universidad de los Andes (Colombia).

** Abogada de la Universidad del Rosario (Colombia), especialista en derecho contractual y magíster en derecho administrativo de la misma Universidad. Magíster en ciencias jurídicas y estudiante de Doctorado en Derecho de la Universidad Pompeu Fabra (Barcelona, España). Ha sido asesora técnica en proyectos de cooperación internacional sobre reformas a la justicia, mejora regulatoria y globalización del derecho. Autora del libro: El rol del juez administrativo colombiano: ¿un actor influyente en las transformaciones del derecho administrativo y en el fortalecimiento del estado social y constitucional de derecho?, próximo a publicar por la editorial de la Universidad del Rosario, y del libro Tendencias internacionales para la mejora de la producción normativa (manuscrito sin publicar). Correo: indiralatorre@gmail.com.
} 
Introducción

La globalización es una de las razones a las que suele acudirse cuando se quiere explicar por qué los Estados incorporan y adaptan sus políticas internas a las tendencias mundiales. Sin embargo, esta explicación no siempre es suficiente para entender por qué esto es relevante, a quién beneficia y qué puede esperarse de su aplicación. El interés por aumentar esta comprensión motivó a tratar en este artículo lo que puede denominarse política regulatoria o política de mejora regulatoria (Better Regulatory Policy).

En el mes de septiembre del año 2013, los países miembros de la Organización para la Cooperación y el Desarrollo Económicos (OCDE) aprobaron la hoja de ruta para que Colombia diera inicio al proceso que le permitirá adherirse a esta organización internacional. Dentro de este plan se señalaron diversos temas que el gobierno colombiano deberá revisar para promover futuras reformas a sus políticas, entre estas, la política regulatoria. ${ }^{1}$

Esta serie de revisiones y de futuras reformas marcan el inicio de nuevos paradigmas para el desarrollo del país en lo político, en lo jurídico y en lo económico. Sería erróneo, o por lo menos algo ingenuo, equiparar este proceso con aqueIlas reformas que surgen como resultado de la celebración de tratados internacionales. En la

1 Con motivo de la adhesión, Colombia también deberá revisar otras políticas, como por ejemplo: las políticas sobre inversiones, gobierno corporativo, medio ambiente, gobernanza pública, economía, educación, trabajo, salud y comunicaciones. adhesión a la OCDE parece haber algo más allá: el llamado a una comprensión diferente de los modos de actuar político y jurídico.

Bajo esta óptica, el presente artículo persigue un doble objetivo: en primer lugar, tiene como propósito introducir en la discusión académica los ejes principales de la política de mejora regulatoria. $Y$ en segundo lugar, pretende resaltar tanto las oportunidades como los desafíos de una política tal en Colombia.

En este sentido, sostendré que la política de mejora regulatoria representa una gran oportunidad para, de manera general, contribuir al Estado constitucional y democrático de derecho en Colombia. Sin embargo, aprovechar esta oportunidad requiere afrontar diversos desafíos. $Y$ un desafío en particular, prioritario respecto a los demás, será el de formular e implementar esta política a partir de una visión amplia de regulación. Esta visión es la clave para entender y afrontar desafíos más concretos, a los cuales me referiré más adelante.

Así, aunque pretendo aportar a la mejora de la comprensión sobre los desafíos de la política regulatoria, mi mayor interés es enfatizar en la necesidad de que Colombia asuma una visión amplia. Pues, en primer lugar, solo bajo esta óptica pueden tomarse en serio los desafíos, y, en segundo lugar, solo así podrán enfrentarse adecuadamente.

Adicionalmente, el planteamiento de estos desafíos puede entenderse como una manifestación de tres tensiones. Dos de ellas se encuentran 
presentes en el estudio de las políticas regulatorias. Así, la primera se refiere a la tensión entre dos visiones de regulación (Prosser, 2010), y la segunda, se expresa en la multidisciplinariedad. Diversas disciplinas han asumido el estudio de dichas políticas, sin que se haya explorado completamente el rol del derecho público en esta área. Por último, la tercera tensión, aunque no es propia de este campo de estudio, surge con motivo de la futura adhesión de Colombia a la OCDE. Se trata de la tensión entre lo estatal y lo no estatal: ¿cómo implementar desde el contexto nacional las tendencias globales?

Para cumplir con el objetivo propuesto, este texto se organiza en dos partes. En la primera, se describe el marco, alcance y significado de la política de mejora regulatoria en el contexto de la OCDE, con el fin de entender los objetivos de esta y sus postulados. En la segunda parte, se hace referencia a las recomendaciones que esta organización ha hecho a Colombia para que diseñe su política regulatoria, y se presentan algunas reflexiones sobre cómo enfrentar los desafíos y aprovechar las oportunidades de esta política.

\section{LAS POLÍTICAS DE MEJORA REGULATORIA}

Hablar de políticas de mejora regulatoria ${ }^{2}$ en el mundo occidental parece ser uno de los efec-

2 En esta sección se utilizará este término en plural porque cada Estado diseña e implementa su propia política. Más adelante se usará en singular para referirse a los postulados de esta política según la OCDE y a la política que próximamente diseñará el gobierno colombiano. tos del cambio que ha sufrido el rol del Estado. Cada vez más, y con mayor consistencia, se afirma que el Estado ha pasado de tener un papel intervencionista a desempeñar un rol regulador (Majone, 1997). Esto es muy importante porque implica un cambio en el modelo de gobernanza, un estilo diferente de intervención del Estado que ha sido asociado con la creación de agencias independientes de regulación. En este nuevo rol, la regulación es la función estelar del Estado, pues es mediante esta que intenta cumplir sus objetivos públicos.

Esta serie de cambios, que pueden agruparse bajo la idea de gobernanza regulatoria, pueden ser entendidos, bien en el ámbito estatal, bien en el no estatal. El primero correspondería, siguiendo a Scott (2010), a la visión asumida, principalmente, por la doctrina norteamericana, la cual tiene como preocupación mayor la naturaleza, alcance y control de la delegación de poder regulador en organismos independientes. Pero esta visión debe complementarse con una visión no estatal. Bajo esta perspectiva, la gobernanza regulatoria no es únicamente un asunto estatal, sino que se encuentra fragmentado entre el Estado y más allá de este (Scott, 2010, p. 21). Organizaciones internacionales, transnacionales, supranacionales, organizaciones civiles internacionales, entre otras, tienen un papel fundamental como creadores de regulaciones (Vibert, 2011). Además, estos actores no actúan solos, sino que unos y otros se sobreponen, incluyendo a los estatales, en las tomas de decisión. De este modo, la noción de Estado regulador parece quedar pequeña ante esta nueva realidad, así que comienzan a proponer- 
se nuevos rótulos para resaltar esta expansión: nodal governance (Shearing y Wood, 2003), post-regulatory state (Scott, 2004), institutional constellations (Jordana y Sancho, 2004) y más recientemente, regulatory space (Vibert, 2014).

Es en este contexto, el de la gobernanza regulatoria, en donde tienen lugar las políticas regulatorias. Muy introductoriamente, estas políticas pueden definirse como aquellas que permiten “determinar cómo se elaboran, evalúan y revisan las regulaciones" (OCDE, 2012, p. 3).

Pero el escenario en donde se ponen en práctica dichas políticas se hace mucho más complejo, dado que no es posible contar con un único significado de regulación. Esta noción ha sido entendida como una forma de intervención del Estado en la economía, como un mecanismo de control social, y como normas con objetivos específicos (Jordana y Levi-Faur, 2004, p. 3). Las diferencias entre conceptos tienen que ver con nuevas perspectivas de análisis. La noción de regulación ha dejado de ser un concepto económico para pasar a ser observada desde distintas perspectivas. Por ejemplo, la regulación también tiene que ver con la modificación de comportamientos (control social) para obtener resultados. Igualmente, la teoría social ha permitido desarrollar diversos enfoques que van más allá de lo económico: la teoría de la autopoiesis o la teoría de la regulación responsable (responsive regulation), entre otras.

Generalmente, en su forma restringida, pero también mayoritaria, se ha entendido como uno de los medios que el Estado utiliza para interve- nir en la economía y equilibrar así las fallas del mercado (Prosser, 2010, p. 1).

Es esta última visión la que interesa resaltar acá porque entra en tensión con una de carácter más amplio. Quienes sostienen una visión amplia han venido observando que a la noción de regulación comienzan a incorporarse objetivos sociales. De acuerdo con Prosser (2010), esta ampliación es una consecuencia de la creación, durante los últimos años, de instituciones con responsabilidades sociales. De modo que los órganos reguladores con funciones económicas ya no son el ejemplo paradigmático de las instituciones reguladoras (Prosser, 2010, p. 3).

Con esta visión amplia de regulación, la importancia de contar con un regulador independiente y con muy poca discrecionalidad se relativiza, pues más que independencia, la búsqueda de objetivos sociales necesita la colaboración del gobierno. Así, se resalta la necesidad de comprender que existen distintas racionalidades en los reguladores, de modo que no solo la racionalidad económica debe servir como centro de los diseños institucionales (Prosser, 2010, pp. 3-5). En este sentido, este artículo hará especial énfasis en variables distintas a la económica, pero esto no significa que la eficiencia económica deje de ser un factor importante, sino que otras racionalidades confluyen y a la vez legitiman las decisiones de los reguladores.

Teniendo en cuenta estas distintas visiones, pareciera que la noción que utiliza la OCDE está planteada en un sentido amplio. Según esta organización, la regulación es determinante para 
el bienestar de las economías y de la sociedad (OCDE, 2012, p. 22), lo que permitiría interpretar que tanto la regulación económica como la regulación social tienen cabida. Por otra parte, su noción no se limita a normas subordinadas provenientes del ejecutivo, sino también del legislativo e incluso de organismos no gubernamentales. Según la OCDE, la regulación

abarca una variedad de instrumentos mediante los cuales los gobiernos establecen requerimientos para empresas y personas. Las regulaciones incluyen leyes, órdenes formales e informales y reglamentos subordinados que emanan de todos los órdenes de gobierno, así como normas procedentes de organismos no gubernamentales o autorreguladores en los que los gobiernos han delegado facultades en materia de regulación (2012, p. 21).

Pero lo anterior no significa que la implementación de las recomendaciones de la OCDE opere con dicha visión. De hecho, es una crítica recurrente señalar que las políticas regulatorias tienen visión centrada en la eficiencia económica (Tala, 2010, p. 199). Sin embargo, lo que interesa resaltar es que en la OCDE parecen existir elementos que sugieren el desplazamiento hacia una visión más abierta.

Afirmar la ampliación del concepto es importante porque gracias a esto se puede observar que se presenta un reflejo o efecto de tal ampliación sobre las reformas regulatorias y, más concretamente, sobre las políticas regulatorias. Reflejo que puede observarse al analizar su evolución.

En este sentido, vale la pena recordar que una de las finalidades iniciales de las reformas re- gulatorias fue la de imponer ciertos límites y requerimientos a los organismos reguladores. Al concebir la regulación como una forma de intervención, y por lo mismo como un elemento dañino para la libertad individual, una de sus primeras preocupaciones fue la de controlar dicha intervención y garantizar al máximo el funcionamiento del libre mercado. Esto permitiría entender el énfasis inicial que se dio sobre el aspecto económico.

Sin embargo, si se mira con detenimiento la evolución de las reformas regulatorias durante los últimos veinte años, puede observarse una progresiva ampliación de sus objetivos.

A finales del siglo XX se ejecutaron diversas reformas regulatorias. Sus orígenes se han situado en 1981 en Estados Unidos. Allí, con la expedición del Decreto Ejecutivo 12291 se impuso a los reguladores elaborar un análisis costo-beneficio de sus proyectos regulatorios, los cuales debían ser evaluados por la Office for information and Regulatory Affairs (OIRA), oficina ubicada al interior de la Office of Management and Budget (OMB). Este periodo de reformas regulatorias puede ser presentado como una consecuencia de los problemas económicos que se atribuyeron a las políticas del bienestar. Dificultades que Ilevaron a plantear una serie de reformas que tenían en su base una idea central: la regulación costaba mucho más de lo que debía y sus beneficios eran muy escasos (Breyer, Stewart, Sunstein y Vermeule, 2006, p. 26).

También en el Reino Unido se dieron este tipo de respuestas, promoviendo no solo el control de la 
regulación, sino la desregulación. Se introduce así una etapa desreguladora, cuyo lema será "menos es más". ${ }^{3}$ Sin embargo, las propuestas desreguladoras tampoco fueron la mejor salida a los problemas económicos que pretendían resolverse. Aparecerán entonces programas de autorregulación y programas de regulación alternativa. Finalmente, comenzará a hablarse de programas de mejora regulatoria (better regulation) y, específicamente, de programas para la mejora de la calidad regulatoria. Esta última etapa es la que, principalmente, se debe tener en mente cuando en este artículo se habla de política regulatoria.

Así, se puede observar un giro que va de la desregulación a la mejora de la regulación. De "menos es más" a "menos o más, pero con calidad". Este periodo de mejora de la calidad regulatoria surge a principios de los años 90 y se ubica en el ideal de la buena gobernanza, en donde la regulación será una parte relevante de los procesos administrativos institucionales. Por ejemplo, en Inglaterra, el cambio de la desregulación a la mejora de la regulación se producirá con la creación de la Better Regulation Task Force (BRTF), así como con la expedición de principios para la buena regulación (Prosser, 2010, p. 206).

El giro hacia la calidad ha supuesto el desarrollo de nuevas técnicas y herramientas en el nivel gubernamental que, incluso, han llevado a afirmar el cambio de Estado regulador al Cost-Benefit State (Sunstein, 1996). Este último, como

3 Este lema fue utilizado en el informe de la Better Regulation Task Force (BRTF, 2005), el cual proponía la reducción de cargas administrativas en el Reino Unido. un Estado que tiende cada vez más a preguntarse por los beneficios de la regulación para, de esta forma, determinar si sus costos son proporcionales y, por ende, justificados.

En todo caso, debe aclararse que la visión amplia en las políticas regulatorias se estaría dando, según Prosser (2010), en el Reino Unido. Así, este autor identifica nuevas áreas en las que la política regulatoria se ha insertado, tales como: los derechos humanos, la equidad y el desarrollo sostenible. Esto lo ha llevado a identificar varios modelos regulatorios, entre ellos, un modelo deliberativo y un modelo basado en valores no económicos, como la protección de los derechos humanos. Por el contrario, una visión restringida en la política regulatoria se limita a la simplificación de trámites administrativos y a la reducción de costos para las empresas (Prosser, 2010, p. 205).

Aunque el análisis del anotado autor está principalmente centrado en el contexto británico, no debe analizarse la tendencia a la ampliación de los modelos regulatorios como casos locales y aislados. Estos cambios también pueden observarse en las políticas de mejora regulatoria de la Unión Europea (UE). Y aunque hasta el momento sea solo una tendencia en desarrollo, ${ }^{4}$ resultará útil reconocer sus beneficios de modo que pueda incentivarse su expansión.

Así, si bien este proceso de evolución muestra una tendencia, no por ello han dejado de existir

\footnotetext{
4 Por ejemplo, Prosser (2010, p. 202) advierte la aproximación restringida de las reformas regulatorias en Australia.
} 
visiones restringidas. $Y$ es que en el fondo se trata de dos lentes para implementar la política regulatoria y crear regulaciones. Incluso en el periodo de la mejora de la calidad regulatoria pueden encontrarse estas dos perspectivas. Una visión restringida defendería una mirada económica sobre la regulación, mientras que la segunda ampliaría esta perspectiva a una dimensión social y deliberativa. ${ }^{5}$

Este breve recuento permite afirmar el reflejo o el efecto de la noción amplia de regulación sobre las reformas regulatorias. $Y$ eso es así, porque la tensión entre ambas visiones es fácilmente trasladable a la política regulatoria. Desafortunadamente, no se trata de un reflejo directo e instantáneo. La voluntad política y la aceptación de una noción amplia de regulación son condiciones necesarias para que la política de mejora regulatoria se formule en términos amplios.

Finalmente, puede advertirse que lo que se ha presentado como una tensión es en realidad una ampliación de la noción de regulación y de la interpretación de las políticas regulatorias. No es que se opongan de modo irreconciliable la eficiencia económica, los valores sociales y la deliberación, sino que la visión amplia adiciona nuevos elementos a los tradicionalmente presentes.

5 Al introducir el ideal deliberativo en la regulación se abre todo un escenario para la discusión sobre la democracia deliberativa, lo cual enriquece el campo de estudio de la regulación. Al respecto, la literatura en esta área ha señalado que la dimensión deliberativa de la regulación debe ir más allá del modelo propuesto por Habermas (Black, 2000).

\section{A. Alcance y significado de la política de mejora regulatoria en la OCDE}

Esta introducción al contexto y a las distintas visiones permite centrarse ahora en el alcance y el significado de la política de mejora regulatoria en el ámbito de la OCDE. A continuación se hará referencia al alcance de la política en términos generales, sin consideración a un contexto institucional en particular, aunque es de suponer que cada Estado diseña su propia política regulatoria según sus correspondientes arreglos institucionales, como de hecho sucede en los países miembros de la OCDE. ${ }^{6}$

Una mirada al desarrollo histórico de las políticas regulatorias muestra que, incluso antes de las recomendaciones de la OCDE, algunos Estados individualmente considerados ya habían iniciado el desarrollo de este tipo de políticas, como es el caso de Estados Unidos o de Alemania. Sin embargo, no puede negarse que la expansión de estas políticas se ha debido, principalmente, a la existencia de actores internacionales y supranacionales como la OCDE y la UE.

La política regulatoria ha pasado por distintas etapas. Primero, se estableció un periodo desregulador, que, posteriormente, fue superado por un periodo de reforma regulatoria (regulatory reform). A este le siguió un periodo de gestión regulatoria (regulatory management), $y$, tiempo después, una agenda de política regulatoria (re-

\footnotetext{
6 La diversidad de políticas puede verse reflejada en los distintos reportes que ha producido la OCDE en el marco del The EU 15 project. Para consultar los reportes ver: http://www.oecd.org/gov/regulatorypolicy/betterregulationineuropeeu15countryfinder.htm
} 
gulatory policy) (Malyzhev, 2006, pp. 275-276). Esta última etapa marca la tendencia actual y permite afirmar que el alcance de la mejora de la calidad de la regulación ha sido cada vez mayor. Esto, porque al reconocérsele el nivel de política, se introduce un conjunto de principios y herramientas para su implementación, con base en los cuales se pretende incidir en todos los organismos reguladores.

Como herramientas de esta política se han señalado: la evaluación de impacto regulatorio (Regulatory Impact Assessment - RIA), la simplificación administrativa y las alternativas regulatorias. Además, se introducen algunos principios y técnicas que pretenden dirigir los procesos de elaboración de normas (rule-making process).

Ahora bien, esta perspectiva amplia no aparece así de explícita en las recomendaciones de la OCDE. Según la Recomendación del Consejo sobre Política y Gobernanza Regulatoria del año 2012, la política regulatoria “define el proceso mediante el cual el gobierno, cuando identifica un objetivo de política pública, determina si empleará la regulación como instrumento de política, y en seguida elabora un borrador y adopta una regulación mediante un proceso de toma de decisiones basado en evidencia" (OCDE, 2012, p. 6).

De la anterior definición no se logra extraer fácilmente el alcance de la política, de ahí que deban tenerse en cuenta otros elementos como el proceso de evolución al que se está haciendo referencia y los principios para la calidad de la regulación, que comentaré posteriormente.
Como se señaló, es posible relacionar las dos visiones de la regulación (restringida y amplia) que propone Prosser (2010) con los distintos periodos institucionales de las reformas regulatorias. De esta manera, con una visión amplia de la noción de regulación, se podría decir que la mejora regulatoria es una política que cobija a todo el Estado, y que incide sobre todos los órganos reguladores en sus distintos niveles (nacionales y supranacionales) para controlar que las regulaciones que se producen o modifican sean de calidad, esto es, vigilando que la regulación fomente el desarrollo no solo económico, sino social y ambiental, a la vez que aumente la deliberación y la legitimidad política de las autoridades regulatorias.

Y puede afirmarse esta visión amplia porque la recomendación de la OCDE del año 2012 incorpora la idea de gobernanza regulatoria señalada al inicio de este artículo. Con este concepto, se busca integrar a diversos actores en el proceso de la elaboración de regulaciones, en todos los niveles y entre todos los sectores, incluyendo, incluso, a los poderes legislativo y judicial. Además, se hace explícito el propósito de entender que la política regulatoria se apropia de una mirada sistémica, definiéndola como una política de gobierno completo (OCDE, 2012, p. 6).

Esta idea de gobierno completo debe asociarse, además, con la noción de transversalidad. Generalmente advertimos que los gobiernos y órganos reguladores participan en la creación políticas públicas: la energética, la ambiental o la sanitaria, esto es, según áreas temáticas. Pues bien, en este caso, la política regulatoria 
sería transversal a todas estas, no es un área temática aislada, sino que debe estar presente en todas y cada una de ellas. Así, dado que en todas las áreas temáticas la regulación se utiliza para cumplir objetivos de política, todas ellas tendrán que sujetarse a la política regulatoria. Esta transversalidad no ha sido suficientemente explorada y vale la pena profundizar si, más bien, de lo que se trata es de una política superior a las políticas temáticas.

De lo señalado hasta el momento se observa que la evolución histórica de esta política muestra un proceso al que cada vez se han ido integrando más y nuevos elementos. Hoy en día, se le debe dar el alcance de política transversal, de gobierno completo, que incluye elementos técnicos y económicos, pero también deliberativos y redistributivos.

Dejando de lado el alcance y definición de la política regulatoria, ahora es relevante tratar de descifrar cuáles son los elementos que la integran y que le dan significado. En este artículo se proponen principalmente dos elementos, aunque se considera que el primero tiene mayor predominio que el segundo. En primer lugar, se observa un elemento procedimental. Y en segundo lugar, un elemento sustantivo. Lo primero supone considerar que con esta política se pretende modelar o guiar el proceso de decisión regulatorio. Lo segundo, en cambio, entiende que esta política direcciona el contenido de la decisión regulatoria.

Pero reconocer la existencia de estos dos elementos puede resultar problemático. A pesar de aceptar que ambos integran esta política, no parece haber suficiente claridad sobre si es el procedimiento el que, en sí mismo, asegura que se obtenga la mejor regulación, o si es un valor sustantivo como la eficiencia económica el que lo asegura, tal y como afirmaría Majone (1997). Una u otra opción trae aparejada una serie de consecuencias y evoca la paradoja entre procedimiento y sustancia. Esto es, que necesariamente se debe aceptar y privilegiar alguno de los dos elementos, ya que, aunque "se impliquen mutuamente pueden entrar en conflicto entre sí" (Martí, 2005, p.154).

El elemento sustantivo es el más controvertido, principalmente porque allí hay un amplio espacio para los desacuerdos y el pluralismo valorativo. Difícilmente podrá llegarse a un acuerdo sobre qué se debería entender por beneficio social, por mejora ambiental, etc.

Además, en el elemento sustantivo se configura un escenario de tensión entre la eficiencia económica y la distribución de beneficios sociales. Casi todos los ciudadanos aceptarían que reducir la contaminación ambiental es un objetivo político innegable, pero seguramente ese nivel de aceptación cambiaría, al menos en algún grado, si, por ejemplo, reducir la contaminación ambiental implica aumentar el costo de producción de los alimentos, ya que esto terminaría por aumentar su valor final. Entonces, ¿cómo se puede determinar la calidad de la regulación? y ¿cuál valor debe primar, la alimentación o el medio ambiente? Estos son desafíos que corresponde resolver en la implementación de la política de mejora regulatoria. Sin embargo, 
pareciera que la mejor forma de entender esta política y resolver estas tensiones es aceptando el predominio del elemento procedimental (Latorre, 2013).

Pero a pesar de esta complejidad, es importante mantener estos dos elementos para entender mejor el significado de la política que acá se comenta. Aunque se tensionen, no se puede negar que están presentes. Es importante, además, porque estos pueden ayudar a construir una interpretación sobre lo que se entenderá por calidad de la regulación

Como se habrá notado, cuando me he referido a la fase actual de las reformas regulatorias lo he hecho utilizando el término "calidad" o "mejora de la calidad". Este término siempre ha estado presente en la mayoría de las recomendaciones de la OCDE, y lo está en la recomendación del año 2012. A primera vista, este concepto no tendría por qué parecer problemático; sin embargo, cuando se intenta abordar el significado de las políticas regulatorias, como es el caso, debe reconocerse que su uso plantea algunas dificultades.

Como adjetivo de la regulación, introduce elementos confusos que no permiten caracterizarla y delimitarla por completo. Como concepto indeterminado, cualquier perspectiva que aborde la noción de calidad la llenará de significados distintos, ya sea desde el área económica, politológica o jurídica. Por ejemplo, desde una perspectiva económica, la calidad puede verse como eficiencia económica y ser este el eje prioritario, pero desde las dos últimas, puede entenderse en un sentido más amplio, como la reunión de otras condiciones adicionales a la eficiencia que otorgan legitimidad política a la regulación (Radaelli y De Francesco, 2007).

\section{B. La evolución de los lineamientos para el desarrollo de la política de mejora regulatoria}

Los anteriores apartados han sugerido que la política de mejora regulatoria es una tendencia global. En todo caso, es importante reconocer que cada país u organización internacional la adopta atendiendo a su propio contexto institucional. Así, aunque no sea nueva en el mundo, quienes lideren esta política en Colombia podrán aprender de las experiencias de estos otros países y decidir cuál es el mejor enfoque que, teniendo en cuenta el contexto político colombiano, puede y debe dársele.

A continuación se hará referencia a las recomendaciones que la OCDE ha elaborado para promover la implementación de la mejora regulatoria en sus Estados miembros. Recomendaciones que, a pesar de no ser vinculantes, tendrían que ser aplicadas en Colombia cuando se ingrese a este selecto grupo de países.

La Recomendación del Consejo sobre Política y Gobernanza Regulatoria del año 2012 tiene sus antecedentes en la Recomendación del Consejo para mejorar la Calidad de la Regulación Gubernamental del año 1995. Esta fue la primera recomendación en la historia que estableció principios para la regulación comunes a varios Estados. Así, establece diez preguntas 
que pretenden orientar a aquellos que toman decisiones políticas y elaboran regulaciones. Estos principios, formulados como una lista de chequeo (checklist), han sido la base sobre la cual se ha seguido construyendo la política regulatoria. Y, en ese sentido, todavía están plenamente vigentes.

Supóngase que el gobierno colombiano planteara, como un objetivo de política ambiental, reducir el impacto socioeconómico del cambio climático. Pues bien, según estos principios, la decisión sobre cómo cumplir con este objetivo debería estar precedida de la respuesta a las siguientes cuestiones:

1. ¿El problema está correctamente definido?

2. ¿Se justifica la acción del gobierno?

3. ¿Es la norma propuesta la mejor forma de intervención del gobierno?

4. ¿Hay una base jurídica para la norma propuesta?

5. ¿Cuál es el nivel de gobierno apropiado para la intervención?

6. ¿Los beneficios de la norma propuesta justifican los costos?

7. ¿La distribución de los efectos de la norma a través de la sociedad es transparente?

8. ¿La norma propuesta es clara, consistente, comprensible y accesible para los usuarios?

9. ¿Todos las partes interesadas han tenido la oportunidad de presentar sus opiniones?
10. ¿Cómo se alcanzará el cumplimiento de la norma? (OCDE, 1995, apéndice).

La anterior recomendación se vio fortalecida en el año 97 con el fin de favorecer la apertura de mercados y la política de competencia. Posteriormente, en el año 2005, se expidió una recomendación con los principios guía para la calidad regulatoria. Esta última recomendación marca el giro hacia la política regulatoria, en cuanto, valga la redundancia, promueve el compromiso político con la mejora de la calidad.

Tanto la recomendación del año 95 como la del 2005 siguen siendo relevantes, solo que ahora la del 2012 hace notable énfasis en el ciclo de la regulación, en la comunicación y coordinación entre todos los actores y niveles de regulación (gobernanza regulatoria) y, como se ha venido diciendo, amplía su perspectiva regulatoria al logro de mejores resultados en lo social, económico y ambiental. Esto, como se señaló anteriormente, amplía el alcance de la política regulatoria.

Es innegable la trascendencia que han tenido estas recomendaciones en los países miembros de la OCDE. En Finlandia, por ejemplo, ya desde la década de los 90 el ministro de Justicia y el ministro de Finanzas recomendaban a todos los redactores de normas seguir la lista de chequeo de la OCDE (Tala, 2010).

Si bien la OCDE ha jugado un rol preponderante en el direccionamiento de las políticas regulatorias, también la UE cuenta con un desarrollo sostenido sobre este tema y, con base en este, dirige todo su proceso de producción norma- 
tiva. ${ }^{7}$ También los países miembros de la UE cuentan con políticas regulatorias. De hecho, 21 países de la UE pertenecen a la OCDE, por lo que existe un notable desarrollo de dichas políticas en estos países. ${ }^{8}$

El informe Mandelkern (2001) fue uno de los elementos base para el desarrollo de esta política en la UE, que además sirvió para enriquecer los planteamientos de la OCDE. Además del informe Mandelkern, la mejora regulatoria en la UE tiene como marco el Libro Blanco sobre la Gobernanza Europea, así como la estrategia para el crecimiento Lisbon Strategy for Growth and Jobs.

Al igual que en el ámbito de la OCDE, en el contexto de la UE la política de mejora regulatoria ha venido evolucionando. Voermans (2009) distingue tres periodos. El primero va desde el año 92 hasta el 2002, y está caracterizado por entender la política de mejora regulatoria como un asunto técnico, enfocado en mejorar la calidad de los borradores de propuestas normativas, así como en su simplificación. ${ }^{9}$ El segundo periodo va hasta el año 2006. Alli la mejora regulatoria

7 Para profundizar el desarrollo de este tema en la ve se puede consultar: http://ec.europa.eu/smart- regulation/index_en.htm

8 Sin embargo, la adopción de esta tendencia global es nueva en Latinoamérica. México ingresó a la OCDE en 1994 y Chile en el año 2010.

9 Según el informe Mandelkern (2001), los antecedentes de la mejora regulatoria también pueden verse desde 1985 cuando se pretendió simplificar la regulación. La simplificación era vista como una forma de intervención del Estado en la economía para alcanzar el establecimiento de un mercado único. La primera inclusión formal de los programas de simplificación se da en el Protocolo Anexo al Tratado de Ámsterdam de 1995 (Mandelkern, 2001, p. 84). Disponible en: http://ec.europa.eu/smart-regulation/better_regulation/ documents/mandelkern_report.pdf es vista como parte integral de la buena gobernanza y se comprende dentro del ciclo de la política pública. Pasan a ser centrales los conceptos de efectividad y eficiencia de la legislación, así como el uso de evaluaciones de impacto de las propuestas normativas. El tercer periodo (2006 hasta hoy) da un paso más, pues incorpora la mejora regulatoria (Better Regulation) como una estrategia general de la UE.

En este último periodo se ubica la Estrategia de Regulación Inteligente ${ }^{10}$ de la Comisión Europea del año 2010, en donde se pone en evidencia la relación entre crisis económica y debilidad de la legislación, y se pretende que, con esta estrategia, se promuevan regulaciones que favorezcan el interés público y el medio ambiente, que impliquen menores cargas administrativas y que permitan el cumplimiento de la estrategia Europa 2020.

A muy grandes rasgos se ha mostrado la evolución de los instrumentos internacionales que han permitido el desarrollo de las políticas regulatorias en los Estados miembros de la OCDE y en la UE. A continuación se hará referencia a algunos de los principios que estas recomendaciones reconocen, con el fin de visualizar las implicaciones que el diseño e implementación de esta política tendrían.

10 Ver: Comisión Europea. (2010a). COM (2010) 543 Final. 


\section{Principios para mejorar la calidad de la regulación}

En el apartado anterior se señaló que desde las primeras recomendaciones de la OCDE se comenzaron a incorporar principios, herramientas y procedimientos que pretendían guiar el ciclo regulatorio. Estos factores fueron esenciales para poner en ejecución la política. Pero sobre todo, porque con su aplicación se esperaba mejorar la regulación, esto es, garantizar su calidad (con la indeterminación que este concepto trae aparejada).

Tal vez hablar de principios no sea lo más exacto porque también han sido vistos como estándares o recomendaciones. Sin embargo, creo que la denominación de principios es apropiada para resaltar su relevancia, al menos, inicialmente, en un sentido pedagógico.

Dado que es imposible agotar acá todas y cada una de las recomendaciones de la OCDE del año 2012, los principios, tal y como fueron presentados en el informe Mandelkern (2001), son un buen punto de referencia para entender los ejes más representativos y novedosos de la mejora regulatoria. En realidad, muchos de ellos son principios que se encuentran en los instrumentos de la OCDE y la UE. Incluso, son similares a los principios que los Estados establecen en el nivel nacional, como es el caso de los Principles of Good Regulation (BRTF, Cabinet Office, 2003) en el Reino Unido.

Contar con principios para la mejora regulatoria es muy relevante, su existencia permite abrir la discusión sobre la necesidad de tener o no un marco para el control de las autoridades regulatorias en un nivel normativo. Esto, para el derecho público, puede ser interpretado como un instrumento de control al poder público. Sin embargo, debe aclararse que no se trata de principios vinculantes, pues su incorporación casi siempre se ha dado en instrumentos de soft law. Cuando son introducidos a través de normas de procedimiento administrativo adquieren fuerza normativa, aunque, algunas veces, allí no logran irradiar el poder normativo necesario para llegar a todas las autoridades regulatorias. ${ }^{11}$

Pero también se genera otra discusión: el uso estricto de procedimientos para regular al regulador ha sido cuestionado por causar la petrificación de la regulación, ${ }^{12}$ afectar la independencia de las autoridades reguladoras y causar retardo desproporcionado en la producción de regulaciones (Shapiro, Fisher y Wagner, 2012, p. 464).

De otro lado, aunque en el ámbito institucional el seguimiento a ciertos principios puede parecer un asunto sin mayores controversias, la literatura ha cuestionado el hecho de que no exista completa claridad sobre cuáles son y cómo se deben interpretar. Así, se ha resaltado la necesidad de comprender a mayor profundidad su significado y la tensión entre estos (Tala, 2010).

\footnotetext{
11 Por ejemplo, Posner y Vermeule (2010) señalan que en Estados Unidos la ley de procedimiento administrativo, aunque aumenta el marco para controlar las actividades de la administración, no llega a controlar las acciones del presidente.
}

12 Por petrificación me refiero al fenómeno conocido como regulatory ossification. Para ampliar esta discusión pueden consultarse: Seidenfeld (1997), Pierce (1995) y Verkuil (1995). 
Adicionalmente, también es importante aclarar el nivel jerárquico que se les otorga, es decir, si son principios normativos o si tan solo llegan al nivel de estándares administrativos no vinculantes para los reguladores. Esta última ha sido la interpretación mayoritaria, aunque no deja de percibirse un esfuerzo por asignarles un sentido normativo. Así, por ejemplo, la teoría del derecho ha buscado asumir un rol en el estudio de los procesos regulatorios. ${ }^{13} \mathrm{Y}$, en el propio campo de la regulación, tanto Sunstein (1990) como Prosser (2010) han formulado principios normativos. Para el primer autor, son principios que pretenden orientar la interpretación de los tribunales sobre regulación económica y social. Para el segundo, son principios dirigidos a los reguladores.

A continuación haré referencia a seis principios, con especial énfasis en lo señalado por el informe Mandelkern. Estos son: necesidad, proporcionalidad, transparencia, accesibilidad, simplicidad y accountability.

Una forma pedagógica de comprender la estructura de estos principios es a través de la noción de ciclo político de la regulación. Este concepto indicaría que el proceso regulatorio inicia con el diseño de una política/regulación y termina con su evaluación. Al traer el ciclo de política pública (diseño, implementación, revisión y evaluación) a la producción de normas, es posible dividir el proceso regulatorio en dos

13 Dentro de estos esfuerzos se ubicaría el movimiento Legisprudence (Wintgens, 2006), como área de la teoría jurídica que cuestiona la validez de las normas y su significado en el sistema jurídico a partir de su creación. fases: una ex ante y otra ex post. En la primera, estaría la definición del problema público y el diseño de la política. Una vez se toma la decisión, y se crea la regulación, inicia la fase ex post de implementación, revisión y evaluación. Esto es útil para entender cómo se ponen en juego los siguientes principios.

\section{Necesidad}

El principio de necesidad opera antes de la implementación de una política. Señala que los productores de normas solo deben proponer una regulación si esta es pertinente para resolver un determinado problema público. Esto presupone, en primer lugar, haber realizado una correcta identificación del problema público y, en segundo lugar, haber considerado que existen otras alternativas, más legítimas y más efectivas, para resolverlo.

En este sentido, parece haber un mensaje en este principio: no siempre la creación de una norma es la mejor manera para resolver los problemas públicos. Pero este mensaje también implica que la norma es vista como parte de los instrumentos para cumplir objetivos de política pública. Esto es, la norma en su función instrumental. ${ }^{14}$

\section{Proporcionalidad}

El principio de proporcionalidad buscaría dar respuesta a la pregunta número seis de la lis-

\footnotetext{
14 Una crítica a la función instrumental del derecho puede verse en Tamanaha (2006).
} 
ta de chequeo señalada arriba. Así, una vez se establezca que la norma es necesaria, según el principio de necesidad, habría que establecer si sus beneficios son mayores que sus costos/ desventajas.

Pero ¿qué se puede entender por beneficios y qué por desventajas o costos? La respuesta a esta pregunta debería tener en cuenta las etapas por las que han pasado las reformas regulatorias, porque en este principio también puede observarse una ampliación de las variables usadas en el análisis de proporcionalidad. De esta forma, ya no está enfocado únicamente en elementos cuantitativos, sino que ha logrado integrar elementos cualitativos.

Un Ministerio de Salud que quisiera promover dentro de su política farmacéutica una regulación para garantizar el acceso a nuevos medicamentos, debería hacer una evaluación no solo del costo de adquisición de estos, o del costo que puede suponer la atención médica de la enfermedad, sino, por ejemplo, de los beneficios en términos del bienestar social conseguido, de los derechos humanos en juego o incluso los beneficios para las generaciones futuras.

Sin embargo, el paso de lo cuantitativo a lo cualitativo no ha sido nada fácil. Señalar esta ampliación del principio de proporcionalidad no implica desconocer que en la práctica carezca de controversia, pues la medición de variables cualitativas no es un tema resuelto. Existen muchas dificultades a la hora de establecer criterios para valorar factores que no son fácilmente cuantificables (Sunstein y Lebrón, 2006;
Sunstein, 2014). Pero a pesar de esto, hay que reconocer que la tendencia es la de promover el análisis de los impactos tanto cuantitativos como cualitativos de las propuestas normativas. La recomendación de la $\operatorname{OCDE}$ (2012, p. 10) establece que las evaluaciones deberán contar con una descripción cualitativa de los impactos difíciles de cuantificar y señala como ejemplos: la equidad, la justicia y los efectos distributivos.

Esta tendencia a la ampliación, de lo cuantitativo a lo cualitativo, también puede observarse en la Comisión Europea, quien ha incluido en el análisis de sus propuestas normativas el impacto en los derechos humanos. ${ }^{15}$ Sin embargo, aunque esta ampliación es valorada positivamente por ir más allá de lo monetario, también se ha cuestionado la manera como ha sido integrado el análisis de los derechos humanos al análisis económico (Toner, 2006). En el mismo sentido, se observa que aunque este principio encuentra un buen nivel de desarrollo tanto a nivel internacional como nacional en la jurisprudencia constitucional, no se ha indagado por completo cuál ha sido la influencia de esta en la aplicación de dicho principio en la mejora regulatoria.

Generalmente, la aplicación del principio de proporcionalidad opera por medio de una herramienta que se ha vuelto esencial en las políticas de mejora regulatoria. Esta herramienta es conocida como evaluación de impacto regulatorio (Regulatory Impact Assessment - RIA) y ha sido resaltada como el punto en donde converge la

15 Esta inclusión se ha dado en el marco de la estrategia para la implementación de la Carta de Derechos Humanos de la Unión Europea. 
neutralidad y la racionalidad de la decisión, junto con la deliberación y la participación.

La RIA es una herramienta clave para lograr la aplicación del principio de proporcionalidad. Aquí también será importante comprender que esta ha tenido un proceso de evolución. Así, si inicialmente fue entendida como instrumento para medir las cargas administrativas, ahora puede concebirse como una herramienta que, dentro de un sistema con ciertos déficits de legitimidad democrática, además de permitir el análisis sobre el equilibrio de los costos para las empresas reguladas, también promueve la deliberación política. Este reconocimiento del elemento deliberativo en las evaluaciones de impacto ha sido reconocido por Sunstein (1996) y más recientemente por Prosser (2010).

No es casualidad que las evaluaciones de impacto hayan concentrado buena parte de la investigación sobre mejora regulatoria (Meuwese, 2008; Radaelli, De Francesco y Troeger, 2008). Sin embargo, la mayor exploración se ha dado desde el campo de la administración pública y muy poco desde el derecho público (Meuwese y Popelier, 2011, p. 457).

\section{Transparencia}

Dentro de este principio se ubican las nociones de participación y consulta. En la medida en que los afectados y los interesados en una regulación puedan participar, esto es, conocer, debatir y retroalimentar sobre una propuesta normativa, se mejora el proceso de conocimiento respecto a un determinado problema, lo que facili- tará el diseño de una regulación más efectiva y también más imparcial.

En este sentido, se espera que el hacer transparente una propuesta normativa ayude a reducir el viejo problema de la captura del regulador. Este principio permitiría enfrentar, en algún grado, la incidencia de los grupos de interés sobre las decisiones de los reguladores. Entre más público sea un proceso, más exigente se vuelve la justificación de decisiones. Así, si se advirtiera que una regulación favorece más a un grupo de interés que a otro, se espera que, al volverse pública, se genere mayor exigencia de justificación por parte de los otros grupos y del público en general, así como de mayores razones y argumentos por parte de los reguladores.

De este modo, este principio promueve la participación y consulta en el momento previo a la toma de decisión. Y es acá en donde reaparece el rol deliberativo de la evaluación de impacto señalado arriba. En algunos diseños concretos de esta política, como en la UE, se prevé llevar a consulta pública las evaluaciones de impacto, con lo cual se estaría aportando mayor información para tomar la decisión y se estaría ordenando o estructurando la discusión sobre los posibles efectos positivos y negativos que traerá la regulación.

\section{Accesibilidad}

Una regulación es accesible si logra ser comunicada adecuadamente y si logra ser conocida por aquellos a quienes afecta. Este principio se sitúa en la fase posterior al diseño de la norma 
(ex post). Es, tal vez, uno de los más conocidos para la disciplina jurídica, porque de allí se derivan consecuencias para el principio de seguridad jurídica y para el Estado de derecho. Correspondería al llamado principio de publicidad en derecho administrativo, y, si se quiere, a uno de los ocho principios propuestos por Lon L. Fuller (1969) que conforman la moral interna del Derecho.

Para llevar este principio a la práctica normalmente se toman prestadas herramientas para mejorar la comunicación, entre ellas, el uso de tecnologías de la información. Por ejemplo, este principio estaría relacionado con la idea de lograr una "base de datos única que contenga todo el acervo regulatorio, información sobre el avance de iniciativas legislativas o regulatorias, y un listado de las enmiendas propuestas" (OCDE, 2013b, p. 3). Pero este es solo un primer nivel. Sería muy poco útil pensar que este principio se cumple únicamente con el acceso a la información. Aunque esto es importante, yendo a un nivel más profundo, no solo se trata de comunicar, sino de dar a conocer la regulación. Estas son nociones relacionadas, pero implican procesos distintos.

Afirmar que un gobierno comunica una norma no significa que la dé a conocer. En este punto es relevante el análisis de Luban (2002), quien tras examinar las decisiones de la Corte Suprema de Estados Unidos, muestra que hoy en día es difícil afirmar que los ciudadanos conocen efectivamente las regulaciones, afectándose o transformándose principios básicos del Estado de derecho. Su investigación lo lleva a afirmar que lo que opera hoy, es un mensaje distinto, ya que los destinatarios de la regulación se deben comportar como si en realidad la conocieran, aunque esto no sea cierto (Luban, 2002, p. 316).

\section{Simplicidad}

De acuerdo con las recomendaciones de la OCDE (2012, p. 12) y el informe Mandelkern (2001, p. 10), este principio busca que la regulación tenga el nivel de detalle necesario para ser entendida fácilmente por los destinatarios.

Con este principio se resalta el valor de la claridad y de lo simple. No solo en términos de garantizar un mejor conocimiento de las normas (relacionado con el principio de accesibilidad), sino para reducir los costos que tiene su cumplimiento e interpretación.

\section{Accountability}

Este principio está planteado en términos de responsabilidad y rendición de cuentas. Para el informe Mandelkern (2001, p. 10), la mejora regulatoria requiere que los destinatarios estén en capacidad de identificar quién es la autoridad pública que originó la regulación.

También supone garantizar que dicha autoridad deba informar sobre las dificultades en la implementación de las regulaciones. Y es que informar las dificultades será parte esencial de la justificación para promover una reforma o una simplificación de la regulación vigente. 
Cuando se habla de reformas a la regulación aparece una herramienta similar a la evaluación de impacto regulatorio, pero no en su perspectiva ex ante, sino ex post. Así, la evaluación ex post de la regulación permite hacer ajustes periódicos, buscando la efectividad de la regulación.

\section{UNA POLÍTICA DE MEJORA REGULATORIA PARA COLOMBIA}

En la primera parte de este artículo traté de describir algunos parámetros de la política de mejora regulatoria en el ámbito de la OCDE, con el fin de establecer una primera idea acerca de su significado, alcance y principios. Igualmente, dentro de esta descripción, puse en evidencia que no hay una única visión para interpretar esta política, pero que, a pesar de esto, las políticas de mejora regulatoria y sus instrumentos, al menos desde una perspectiva conceptual, han ido evolucionando hacia modelos deliberativos y redistributivos, y hacia modelos donde muchos actores, no solo las agencias de regulación, convergen.

Ahora, en esta segunda parte, traeré los anteriores elementos al caso colombiano, con la intención de plantear oportunidades y desafíos en el diseño futuro de esta política. En primer lugar, haré una breve referencia al estudio que la OCDE (2013a) hizo sobre Colombia, con base en el cual realizó recomendaciones para que el país formule su propia política. A partir de estas recomendaciones, y de lo que he señalado en la primera parte, identificaré los puntos de partida que permiten realizar una interpretación amplia para formular la política regulatoria en Colombia, así como los desafíos para la investigación jurídica, la democracia deliberativa, el diálogo interinstitucional, la racionalidad e imparcialidad de las decisiones y la cultura jurídica y política.

\section{A. Las recomendaciones de la OCDE}

Si se preguntara por las razones que llevan a que Colombia adopte el discurso de la política regulatoria, podría decirse que existen ciertas condiciones institucionales que le permiten acogerlo. Básicamente, se trata de la institucionalidad que plasmó la Constitución Política de 1991, en donde el rol del Estado se centró en controlar y supervisar, y no en prestar o ejecutar servicios. Este rol comenzó a tomar mayor forma con la creación de comisiones de regulación y superintendencias, así como con la expedición de marcos regulatorios que pretendieron irradiar seguridad jurídica y transparencia en la prestación de los servicios. Pareciera entonces que desde hace más de dos décadas existe un discurso nacional receptivo a la tendencia global de una política regulatoria. ${ }^{16}$

16 Esta receptividad no lleva a afirmar que la política regulatoria puede ser implementada de la misma manera como se ha hecho en países desarrollados. Así, toman importancia los estudios que pretenden explicar el funcionamiento del Estado regulador en los países en desarrollo. Recientemente, ha comenzado a surgir una literatura que, entre otras cosas, ha observado que el rol de los tribunales constitucionales y del poder legislativo en el control de la regulación es distinto del rol asumido en los países desarrollados (López-Murcia, 2013; Dubash y Morgan, 2012; Urueña, 2012). 
A partir de esta base institucional, la OCDE diagnosticó tanto los problemas como los puntos favorables para implementar una política como esta en el país. En términos generales, el estudio observó que Colombia aún no convive con una política de gobierno sobre calidad normativa (2013a, p. 16).

Aunque la OCDE encontró una serie de iniciativas aisladas, las valoró como puntos favorables para una futura formulación de la política. Dentro de estas iniciativas están, por ejemplo, el trabajo adelantado sobre técnica normativa (contemplado en el Decreto 1345 de 2010), el deber de información sobre los proyectos de regulación (art. 8 de la Ley 1437 de 2011), la creación de ventanillas únicas para la simplificación de trámites empresariales, la política de competencia económica, y la obligación de consulta a la Superintendencia de Industria y Comercio (OCDE, 2013a, p. 39).

Pero también se encontraron elementos problemáticos. El diagnóstico observó que diversas autoridades se encargan de asuntos relacionados con la política regulatoria, de manera que existe riesgo de redundancias y duplicidad de esfuerzos. Dentro de estas instituciones están: el Departamento Nacional de Planeación, el Ministerio de Tecnologías de la Información y las Comunicaciones, el Departamento Administrativo de la Función Pública, el Ministerio de Justicia y del Derecho, las comisiones de regulación y las superintendencias (OCDE, 2013a, p. 15). Este aspecto es importante porque desde sus primeras recomendaciones la OCDE siempre ha insistido en la necesidad de contar con una úni- ca institución responsable de promover la política regulatoria en la Administración. ${ }^{17}$

También se señaló que no existen estándares para la preparación de regulaciones, y que apenas se tiene una experiencia muy limitada sobre el uso de herramientas de evaluación de impacto ex ante en la toma de decisiones. Reconoce que hay algunos avances, como el Estudio de Impacto Normativo que los ministerios y departamentos administrativos deben hacer cuando preparan decretos y resoluciones que serán firmados por el presidente. 0 la evaluación de impacto ex ante que, de acuerdo con el Decreto 2696 de 2004, deben hacer las comisiones de regulación (OCDE, 2013a, p. 95). Igualmente, se afirmó que Colombia no cuenta con experiencia suficiente en la elaboración de evaluaciones ex post de leyes y regulaciones (OCDE, 2013a, p. 18).

El completo y exhaustivo diagnóstico que aparece en estas recomendaciones permite justificar las tareas que actualmente tiene Colombia para crear una política regulatoria. Así, se espera que el país formule una política explícita de mejora de calidad regulatoria, con procedimientos, principios, instituciones y herramientas. Además, el mismo documento de recomendaciones sugiere ir más allá de la simplificación de trámites y abordar la calidad de la regulación en un sentido integral.

De todas las recomendaciones que hizo la OCDE (2013a), vale la pena resaltar aquellas vincula-

\footnotetext{
17 La institución emblemática ha sido la Oficina de Información y Asuntos Regulatorios (OIRÁ, por sus siglas en inglés) que es parte de la Oficina de Administración y Presupuesto, la cual se encuentra al interior de la Oficina Ejecutiva del presidente de los Estados Unidos.
} 
das con los desafíos que se señalarán más adelante: la creación de un órgano de supervisión que se haga cargo de la política regulatoria; la mejora de los mecanismos de coordinación entre ministerios, comisiones de regulación y superintendencias; la integración de evaluaciones de impacto para apoyar la formulación de políticas; y la construcción de estándares y requisitos administrativos para elaborar normas.

Vale la pena tomarse en serio estas recomendaciones porque así se comenzará a producir un cambio estructural en las instituciones colombianas que, a su vez, llevaría a modificar conductas en los creadores de normas, y en un nivel más general, a modificar la percepción sobre la cultura de la legalidad. Este no es un cambio instantáneo, pero lo importante será iniciar con una visión adecuada sobre lo que implica esta política. Visión que, como se ha sugerido en este artículo, debe hacerse en términos amplios.

\section{B. Una hoja de ruta con desafíos y oportunidades}

Desde el inicio he estado señalando que, como sucede con la mayoría de tendencias globales, construir una política de mejora regulatoria en Colombia traerá desafíos y oportunidades. A partir de estas recomendaciones y de lo señalado en la primera parte de este artículo pueden establecerse algunos desafíos y oportunidades no solo institucionales, sino también académicos.

La oportunidad mayor será el aporte que la formulación e implementación de la política en su conjunto tendrá para el fortalecimiento del Estado constitucional y democrático de derecho en Colombia. En principio, puede parecer extraño que se vinculen las nociones del Estado constitucional con las del Estado regulador, pues alguien podría esperar que este modelo de gobernanza se relacionara con conceptos sobre todo económicos, como la competitividad y el equilibrio económico. No pretendo negar las ventajas que esta política podrá representar en el nivel económico, mi preocupación se centra en explorar otro lado de esta política, el lado que se ha estado relacionando con la noción amplia de regulación.

De esta manera, como se afirmó desde el inicio, aprovechar esta oportunidad requiere afrontar diversos desafíos. Y uno en particular, que condiciona a los demás, será el de formular e implementar esta política a partir de una visión amplia de regulación. Esta visión será la clave para entender y afrontar otros retos que tienen que ver con la investigación jurídica, la democracia deliberativa, el diálogo interinstitucional, la racionalidad e imparcialidad de las decisiones y la cultura jurídica y política.

Los entiendo como desafíos porque suponen plantear preguntas y enfrentar problemas tanto conceptuales como prácticos. Si se superan, la oportunidad se hará más clara. Si el gobierno y la academia colombiana intentan dar respuesta a las preguntas y se enfrentan adecuadamente a los problemas, entonces las ventajas de la política regulatoria se harán más visibles. 


\section{Ampliar la visión sobre la regulación}

Este es un desafío general, y afrontarlo es una condición necesaria para poder tomarse en serio y enfrentarse a los otros retos.

Del conjunto de las recomendaciones formuladas por la OCDE al gobierno colombiano puede observarse el propósito de corregir la aproximación restringida que algunos de sus países miembros han dado a esta política, estancándose en la simplificación administrativa y en la reducción de cargas burocráticas. Las últimas recomendaciones de esta organización internacional promueven una política de gobierno completo, que además de simplificar y reducir costos, mejore la cooperación y coordinación entre todos los sectores y actores que participan en el proceso regulatorio (OCDE, 2012, p. 8). En esta medida, se fomenta una ampliación que va de la simplificación administrativa a una concepción integral y sistémica del proceso regulatorio.

Como he venido afirmando a lo largo de este artículo, la ampliación de la política está relacionada con la noción de regulación, y por eso he tratado de defender la visión de regulación que propone Prosser (2010) como el lente que permitirá formular la política de mejora regulatoria en Colombia. Sin embargo, como la noción que propone este autor se origina en la racionalidad de las distintas agencias de regulación y en el desarrollo de las reformas regulatorias en Inglaterra, ¿con qué elementos cuenta Colombia para adoptar una visión amplia? Alguien podría afirmar que esta es una visión anglosajona que no responde a la realidad institucional, y que
Colombia tiene que iniciar asumiendo una postura restringida para luego, a partir de su propia evolución, ampliar la perspectiva.

Pero, aunque Colombia esté iniciando el proceso de conocimiento sobre la política de mejora regulatoria, ello no implica que carezca de un proceso de evolución sobre la noción misma de regulación. Por un lado, se ha venido afirmando que la evolución de noción de regulación se refleja en las políticas de mejora regulatoria, y en ese sentido, sería de esperar que Colombia asuma este reflejo e incorpore una noción amplia de regulación. Pero por otro lado, es bastante relevante la investigación que hace Urueña (2012), quien demuestra que la concepción amplia de regulación se ha insertado en Colombia por medio de la interpretación judicial, en donde la Corte Constitucional ha incluido variables como la protección de los derechos humanos al proceso regulatorio.

Esto permite afirmar que el gobierno colombiano cuenta con dos incentivos fuertes para enfrentar el desafío: una tendencia global y una tendencia nacional.

Con este enfoque parece posible formular una política regulatoria en donde se hagan explícitas las ideas afirmadas a lo largo de los anteriores epígrafes: que mire hacia otros valores (no solo la eficiencia económica), y que promueva la deliberación política y el diálogo interinstitucional. 
2. Promover la investigación jurídica sobre mejora regulatoria

La visión puramente económica o restringida de la regulación y de las políticas regulatorias ha ocasionado que el desarrollo de la investigación y discusión académica desde áreas como la jurídica o la politológica (al menos en Europa) no presente avances más profundos e innovadores sobre las políticas de mejora regulatoria (Radaelli, 2007; Brown y Scott, 2011).

Distintas áreas de estudio han abordado el tema de las políticas regulatorias, de hecho, se trata de un área en donde varias disciplinas han desarrollado campos propios de investigación, como la ciencia política y la economía. Pero no ha sido usual que se explore desde el derecho público (Brown y Scott, 2011, p. 476). Sin embargo, como se ha visto, la idea de contar con una política regulatoria sugiere categorías de análisis importantes para abordar los límites al poder público, de modo que podría construirse una agenda para la investigación jurídica sobre esta política.

En primer lugar, la noción de ciclo político de la regulación, ajena a la estructura tradicional del pensamiento jurídico, puede abrir un nuevo campo de investigación. Tradicionalmente, el área jurídica ha estado enfocada en estudiar la fase ex post de la regulación. Ahora, con la mejora regulatoria se debe dar una mirada a la fase previa de la expedición de una norma. De esta manera, con la noción de ciclo político reaparecerán cuestiones que para el positivismo jurídico parecían resueltas.
En consecuencia, será relevante indagar cómo el proceso de elaboración de determinadas políticas incide sobre el proceso de elaboración de normas, lo cual termina por acercar el mundo jurídico al mundo político. Se trataría, entonces, de explorar nuevos modelos de investigación jurídica. Modelos como el propuesto por Biernat (2008), que se caracterizan por examinar la relación entre policy y law making. El escenario más usado para explorar este enfoque de investigación es la UE, pero esto no impide estudiar otros escenarios. En esta organización, por ejemplo, la legitimidad de las decisiones y actividades políticas son vistas como un complemento de la legitimidad del derecho creado, de modo que investigar sobre los sujetos, su legitimidad, su proceso de razonamiento y los principios que deberían guiar las decisiones, es algo necesario para comprender y cuestionar la legitimidad del derecho que se produce (Biernat, 2008, p. 98). Este enfoque resultaría muy enriquecedor para el desarrollo de la mejora regulatoria en Colombia.

En segundo lugar, la evolución de las políticas regulatorias hacia modelos deliberativos y sociales, así como la idea de una mejor calidad de la regulación, deberían servir de motivación para que la investigación en derecho público aporte a una política que, en principio, bajo una visión restringida, podría ser solo de interés para economistas o administradores públicos. De hecho, se vuelve todavía más relevante porque, como muestra Radaelli (2007), no siempre ha sido posible probar con certeza los beneficios que las políticas de mejora regulatoria han traído para el crecimiento económico. 
En tercer lugar, la política de mejora regulatoria se apoya en ciertos estándares procedimentales para las autoridades regulatorias, esto es, guías sobre cómo se deben pensar, construir y redactar las normas. Estos estándares se configuran, entre otros, sobre dos grandes presupuestos: la participación de interesados y afectados con las decisiones políticas, y la toma de decisiones fundadas en la evidencia. A pesar de esto, no existen suficientes estudios sobre lo que implica, para el derecho, contar con estándares y requisitos administrativos para la preparación de regulaciones. Tampoco está claro cuál es la relación de estos principios con los principios para la legislación o los principios del derecho.

Además, estos principios apuntan a lograr la calidad regulatoria, pero ¿qué puede entenderse por calidad desde una perspectiva jurídica? En este sentido, también será importante el aporte jurídico en la formulación de criterios para medir la calidad de la regulación. Esta es una tarea que desde el año 2004 comenzó a realizar la UE, estableciendo una serie de indicadores que han tratado de ir más allá de la eficiencia regulatoria. ${ }^{18}$

Estas son razones importantes para que la investigación jurídica se entusiasme por hacerle seguimiento a esta política, y para que contribuya a resolver o plantear inquietudes que, seguramente, generará su implementación.

En el ámbito europeo también se han planteado algunas inquietudes. Por ejemplo, si los princi-

18 Ver la descripción del proyecto en: http://www.brad.ac.uk/irq/project/ pios o estándares intentan evadir los límites que señala el derecho constitucional y administrativo para la creación de normas, o si el poder judicial tiene en cuenta los estándares y las evaluaciones de impacto ${ }^{19}$ y si son instrumentos que, efectivamente, permiten mejorar el control de la legislación delegada. También se ha propuesto discutir el nivel de obligatoriedad de los estándares y considerar sus posibles efectos jurídicos (Meuwese y Popelier, 2011).

En definitiva, la mejora regulatoria no debería escapar del análisis jurídico, ya que puede ser entendida como una política en donde el derecho es quien se encarga de regular su propia mejora (Betancor, 2009, p. 20).

\section{Emprender el camino hacia la democracia deliberativa}

Se presenta un desafío en el elemento deliberativo porque introduce a Colombia en un modelo de democracia que no ha sido completamente explorado por las instituciones públicas y la sociedad civil. La democracia representativa y, en los últimos años, la participativa, han tenido más juego en la vida pública colombiana.

La democracia deliberativa llama al estudio de teóricos vinculados a este modelo, tales como Jürgen Habermas, Philip Pettit, Bernard Manin, Joshua Cohen, entre otros. La democracia deliberativa "es un modelo político normativo cuya propuesta básica es que las decisiones políticas

19 Por ejemplo, en Estados Unidos, los tribunales revisan activamente las evaluaciones de impacto regulatorio. 
sean tomadas mediante un procedimiento de deliberación democrática" (Martí, 2006, p. 22).

Entender la producción normativa en este marco democrático implicará dos elementos. Primero, que las decisiones deberían ser tomadas con una amplia participación de aquellos que se verían afectados con la regulación futura, y segundo, que este escenario participativo supone debatir y argumentar las distintas posiciones sobre la regulación propuesta. Es decir, este modelo combina democracia y deliberación (Martí, 2004, p. 22-31).

Generalmente, este elemento deliberativo ha sido llevado a la práctica regulatoria a través del principio de proporcionalidad que, por un lado, permite considerar los costos/beneficios de la regulación, y, por otro lado, aportar razones para que los reguladores y actores políticos tomen decisiones justificadas e informadas. Es en este punto en donde aparece el factor deliberativo, pues parte de esas razones es obtenida a través de un proceso de deliberación. Así, es importante que la regulación refleje los juicios deliberativos y que evite ser el resultado del poder de los grupos de interés, la falta de información, el poder de los medios de comunicación, etc. (Sunstein, 1996, p. 22).

Herramientas como las evaluaciones de impacto, en donde se llevan a cabo análisis costobeneficio, deberían interpretarse en un marco democrático deliberativo. Esto implica entender que, bajo evidencia empírica, podrá expedirse una regulación que, incluso resultando más costosa, sea la acordada por todos los afectados.
Con el trasfondo deliberativo, las evaluaciones de impacto regulatorio permiten disminuir la presión de los grupos de interés sobre el regulador y conseguir que los impactos de una regulación sean conocidos y sometidos a debate de los ciudadanos (Sunstein, 1996, p. 4).

Este es quizás el mayor desafío, que incluso ha sido difícil de enfrentar en otros países con democracias más fuertes. Por ejemplo, en Finlandia se ha cuestionado que los programas de reformas regulatorias han respondido al modelo de elección racional, el cual se enfoca en superar los problemas de información sin integrarse a sociedades con pluralidad de ideas. Esto ha llevado a plantear el denominado institutional realistic-model, caracterizado, entre otros elementos, por la existencia de desacuerdos y la influencia de diversas comunidades o grupos (epistemic communities) en la elaboración de normas (Tala, 2010, p. 209).

Hacia este ideal debería apuntar la formulación de la política en Colombia. Claramente, la puesta en práctica de un proceso deliberativo también requiere un adecuado tratamiento de los procesos de participación democrática. Pero no como un fin, sino como uno de los elementos que permite acercarse al ideal deliberativo. En este sentido, serán esenciales las herramientas para mejorar la transparencia y participación durante el proceso de decisión. Solo gracias a la información se podrá opinar, responder y escoger entre distintas alternativas regulatorias. Sin embargo, no hay un entendimiento unánime sobre las implicaciones de la transparencia en los procesos regulatorios. Las doctrinas del public 
management han formulado distintos diseños de transparencia regulatoria: Fiduciary trusteeship, consumer sovereignty y empowered citizenship. Cada diseño provee herramientas distintas para concretar su modelo. Los dos primeros son caracterizados como procesos de decisión elitistas, mientras que el último promueve la participación de los ciudadanos, reduciendo la distancia entre los afectados y los reguladores (Lodge, 2004, p 130-132).

En esta vía, aparece un largo camino por construir y muchas preguntas por resolver. Pero en todo caso no se debería desconocer que una política de mejora regulatoria que tenga como perspectiva el ideal deliberativo, mejorará la calidad de la democracia y asegurará una mayor legitimidad de las decisiones regulatorias.

\section{Fortalecer la búsqueda de decisiones razonadas e imparciales}

Alcanzamos un mayor grado de imparcialidad en las decisiones cuanto mayor y mejor ha sido el proceso de deliberación (Gargarella, 1998). Sin embargo, en la política de mejora regulatoria también hay otra forma de imparcialidad. Esto es así porque el ámbito de la regulación nos pone en el nivel de decisiones que requieren un grado de experticia.

Así, quien toma decisiones basadas en razones empíricas alcanza otro grado de imparcialidad. Pero ¿cómo se concreta este nivel de imparcialidad en la política regulatoria? La respuesta se encuentra en las evaluaciones de impacto regulatorio (RIA).
Sin embargo, varios desafíos parecen ineludibles. Esta herramienta puede asumir versiones y objetivos muy diversos según el contexto institucional en el cual se aplique. Será diferente para la UE y para cada país que incorpore las recomendaciones de la OCDE. Además, aunque los resultados de estas evaluaciones provean información basada en la evidencia y permitan tomar una decisión más informada, cada actor filtrará dicha información a través de marcos cognitivos distintos (Radaelli, 2007, p. 43). Esto permite advertir que los desacuerdos sobre las decisiones no solo se dan en materia de valores, sino sobre el entendimiento del problema público mismo, esto es, sobre la interpretación de la evidencia empírica.

Esta pluralidad de visiones hace que la RIA termine por inclinarse más hacia lo político que hacia lo técnico, y que deje de ser vista como una herramienta de imparcialidad científica pura. De ahí que haya asumido distintos usos: en Italia solo es utilizada para la formulación de algunas políticas; en Francia no antecede el proceso político, sino que se utiliza una vez alcanzado el acuerdo político, jugando un rol post decisional (Radaelli, 2007, p. 45); en la UE la evaluación de impacto es presentada por la Comisión al tiempo con la propuesta de regulación, sirviendo de guía y base para la coordinación del diálogo entre actores (sirve para la articulación del lobby y la formulación de la política).

Lo anterior muestra un gran reto no solo en el aprendizaje de la herramienta por parte de la administración pública, sino en el uso y nivel que se decida dársele. 
Vale la pena resaltar iniciativas recientes como la creación del Centro de Altos Estudios Legislativos (CAEL). Aunque surge como propuesta del poder legislativo, permite atribuirle el interés por fortalecer la evidencia empírica de las decisiones. Creo que esta iniciativa llega en el mejor momento a Colombia, pues le debería permitir, entre otras cosas, insertarse en la lógica de las políticas de mejora regulatoria y mejora legislativa desde el inicio.

Aunque en este artículo no me he referido al papel esencial que desarrollan los parlamentos en el control de las agencias regulatorias, debe anotarse que hoy en día algunos, incluyendo el Parlamento Europeo, han dispuesto comisiones o unidades propias que evalúan el impacto de sus propuestas o de las de otros poderes públicos. Los debates parlamentarios se organizan alrededor de los documentos que contienen las evaluaciones de impacto, logrando así una discusión informada y con evidencia científica.

Tal vez, tomando experiencias extranjeras, el cael pueda asumir un rol especial en la política de mejora regulatoria, que permita pensar en la creación de un tipo de instituciones o unidades similares para que enriquezcan con argumentos técnicos las propuestas normativas de las agencias de regulación y las del propio poder legislativo. No quiero sugerir con esto un diseño institucional en particular, sino llamar la atención sobre la importancia de fortalecer la evidencia empírica de las propuestas normativas.

\section{Mejorar el diálogo entre poderes y sectores administrativos}

Este desafío supone incorporar y poner en práctica la idea de la política de mejora regulatoria como política de gobierno completo.

No debe perderse de vista el rol del poder legislativo. En primer lugar, porque el parlamento es parte del proceso regulatorio, como elemento de control político, y como creador de normas. El concepto de regulación que tiene la OCDE es amplio, esto es, incluye actos del ejecutivo, de organismos independientes reguladores y del parlamento. ${ }^{20}$ Por consiguiente, será importante reflexionar sobre la calidad de la legislación, y sobre si los principios para la calidad de las normas provenientes de las comisiones de regulación y del poder ejecutivo también deberían guiar el proceso de producción de leyes.

El rol del parlamento, al menos en países con democracias fuertes, es el de ejercer control sobre los proyectos de regulación de iniciativa del ejecutivo o de otras autoridades regulatorias. Esta es una función determinante para el Estado de derecho y el principio de división de poderes. A pesar de ello, en Colombia esta función no se cumple adecuadamente (López Murcia, 2013 , p. 124), aspecto que crea mayores desafíos para la política regulatoria, dado el carácter de gobierno completo promovido por la OCDE.

20 El concepto de regulación de la OCDE también incluye "normas procedentes de organismos no gubernamentales o autorreguladores en los que los gobiernos han delegado facultades en materia de regulación" (2012, p. 21). 
Por otra parte, también resulta desafiante el rol del poder judicial en esta política. En primer lugar, porque como demuestra López Murcia (2013), el papel de las Cortes constitucionales frente a la regulación en países en desarrollo es distinto, y esto hará que deba reflexionarse sobre cuál será su función en la política regulatoria. Por ejemplo, la jurisprudencia podría enriquecer la interpretación de los principios o estándares a los que se hizo referencia en la primera parte de este artículo. Pero además, estos principios también podrían nutrir la interpretación y argumentación judicial, junto con elementos provenientes de las evaluaciones de impacto. Es decir, la política regulatoria entregaría a los jueces la mirada previa (ex ante) a la expedición de la norma, ya no limitada a la exposición de motivos, sino a otros instrumentos como las evaluaciones de impacto y a los documentos que reflejen los procesos de consulta. Si en Colombia las Cortes han jugado un papel clave para entender el Estado regulador (Urueña, 2012), este todavía podrá acentuarse más bajo una política de mejora de la calidad regulatoria.

Adicionalmente, los desafíos tres y cuatro muestran la imposibilidad de que la RIA sea una herramienta de imparcialidad pura, aislada del debate político, de ahí que también adquiera importancia como instrumento de diálogo interinstitucional. Un desafío será convertirla en un instrumento de diálogo articulado entre sectores administrativos y poderes públicos.

Finalmente, y aunque este tema daría para una nueva investigación, es muy relevante el diseño institucional que se formule para poner en funcionamiento el órgano encargado de revisar y coordinar la elaboración de las regulaciones. Este parece ser el objetivo tras la reciente comunicación sobre la reestructuración de la Presidencia de la República. ${ }^{21}$

\section{Revaluar la cultura jurídica y política}

Aunque el concepto de cultura jurídica es bastante ambiguo, con ello me refiero, tomando a Friedman (1994, p. 118), a las ideas, valores, actitudes y opiniones de las personas con respecto al derecho y al sistema jurídico. Es difícil negar que el sistema jurídico colombiano cuenta con bajos índices de favorabilidad, o que la cultura de la legalidad tiene bastantes deficiencias. Colombia tiene una cultura del uso simbólico del discurso legal, en la cual "el sistema jurídico se convierte más en un mecanismo destinado a la legitimación de las políticas públicas que en un instrumento de implementación instrumental de dichas políticas" (García Villegas, 2002, p. 32).

Como afirmé al inicio de este texto, la adhesión de Colombia a la OCDE podría representar nuevos modos de actuar jurídico. Se ha visto que los principios y herramientas de la política regulatoria podrían aumentar los procesos deliberativos y la transparencia en los procesos de decisión, también podrían generar decisiones más imparciales fundadas en evidencia empírica. Sin duda, si se logra esto, entonces las normas contribuirán a la solución efectiva de los problemas

21 Ver: http://www.semana.com/nacion/articulo/santos-reforma-estructura-de-presidencia-de-la-republica/399047-3 
públicos, evitando que intereses individuales, electorales y económicos se confundan en las iniciativas regulatorias.

En Colombia se ha evidenciado una abundante expedición de normas. Durante el periodo 2007 y 2012 se expidió en promedio un decreto cada medio día, y durante el 2002 y 2012 una ley cada cuatro o cinco días (Departamento Nacional de Planeación, 2013). Además, durante el periodo 2010-2014, ocho de cada diez leyes fueron archivadas (Morelo, Báez y Garibello, 2014). Estos datos son una razón más para creer que Colombia merece tener una política regulatoria, porque esto ayudaría a planear y evaluar, conscientemente, datos como los anteriores.

Igualmente, contar con una política de mejora de la calidad regulatoria debería llevar a considerar relevante la profesionalización de la redacción de leyes y regulaciones, lo cual implica seguir el principio de accesibilidad y simplicidad, y entender su redacción dentro de un proceso de creación de políticas y normas.

Igualmente, una implementación adecuada de la política regulatoria supondrá desafíos para los modos de actuar políticos. La institucionalización de los procedimientos, herramientas y principios implicará mejorar los vínculos entre representantes y ciudadanos. Mejorar la comprensión sobre los problemas de la representación política es fundamental para que la política de gobierno completo cumpla su finalidad, pues, con la perspectiva optimista que se asume acá, la política regulatoria necesita un mayor compromiso por parte de los representantes políti- cos y agentes reguladores para trabajar en la solución de problemas públicos (Rey Salamanca, 2014).

\section{CONCLUSIONES}

El propósito central de este artículo ha sido introducir en la discusión académica los principales elementos que conforman la política de mejora regulatoria. Se ha señalado que la formulación de una política regulatoria debería tener en cuenta otras variables, distintas a la eficiencia económica y la competitividad. Variables como la equidad, la deliberación, los derechos humanos, los efectos distributivos de las decisiones regulatorias, etc. Estas nuevas variables responden, en primer lugar, a una visión amplia de la noción de regulación, la cual ha sido propuesta por autores como Prosser (2010). En segundo lugar, se encuentran relacionadas con el rol que la Corte Constitucional ha jugado en las decisiones regulatorias (López Murcia, 2013; Urueña, 2012), y finalmente, estas variables surgen de la evolución de las recomendaciones de la OCDE y de los principios para la mejora regulatoria. En definitiva, Colombia no está para reproducir miradas restringidas.

Este artículo ha sostenido una perspectiva optimista de la política de mejora regulatoria, señalando oportunidades y desafíos de su implementación. El desafío más importante, y por lo mismo prioritario, es el de incorporar la noción amplia de regulación en esta política. Esto es, incluir las anteriores variables, pero también comprender que la regulación va mucho más 
allá de la simple creación normativa y que se trata de una política de gobierno completo, en donde múltiples actores y niveles de gobierno entran en juego.

Esto es prioritario porque es la base para tomarse en serio los otros desafíos. Así, solo con una visión amplia adquiere relevancia el reto de promover la investigación jurídica sobre mejora regulatoria, de emprender el camino de la democracia deliberativa y de mejorar la participación. También de fortalecer la búsqueda de decisiones razonadas e imparciales, de mejorar el diálogo entre poderes y sectores administrativos, y finalmente, de revaluar la cultura jurídica y política en Colombia. De esta manera, si se llegara a crear una agenda política y académica enfocada en todos estos desafíos, entonces se harán notorias las contribuciones para el Estado constitucional y democrático de derecho. Por eso, como se ha señalado, el fortalecimiento de este modelo de Estado es la mayor oportunidad de la política de mejora regulatoria.

Como señalé, se ha asumido una posición optimista, pensando que es posible tener una adaptación adecuada y satisfactoria de esta política. Pero se ha hecho en términos generales. Es decir, se reconoce que existen particularidades institucionales que requieren una mayor elaboración de la que se ha podido presentar acá.

En este sentido, la formulación de la política debe hacerse teniendo siempre presente la siguiente pregunta: ¿cómo debe ser diseñada esta política teniendo en cuenta el contexto colombiano? Para responder a ello resulta muy apropiada la nueva literatura sobre el Estado regulador en los países en desarrollo (López Murcia, 2013; Urueña, 2012; Dubash y Morgan, 2012), la cual propone nuevas formas de análisis, distintas a las usadas en países desarrollados. Seguro que este nuevo enfoque podrá realizar aportes significativos para la formulación de la política de mejora regulatoria en Colombia.

\section{Referencias}

Betancor, A. (2009). Mejorar la regulación: una guía de razones y de medios. España: Marcial Pons, Ediciones Jurídicas y Sociales.

Better Regulation Task Force. Cabinet Office. (2003). Principles of Good Regulation. Obtenido de BRTF: http://webarchive.nationalarchives.gov.uk/20100407162704/http:/archive.cabinetoffic e.gov.uk/brc/upload/assets/ www.brc.gov.uk/principlesleaflet.pdf

Better Regulation Task Force. (2005). Regulation-Less is More: A BRTF Report to the Prime Minister. Recuperado de http://www. policy.manchester.ac.uk/media/projects/policymanchester/civilservant/les s-is-more.pdf

Biernat, T. (2008). Law making policy. In T. Biernat, M. Zirk-Sadowski y M. Kieltyka, Politics of law and legal policy: between modern and post-modern jurisprudence (págs. 75-158). Kraków: Wolters Kluwer Polska.

Black, J. (2000). Proceduralizing Regulation: Part I. Oxford Journal of Legal Studies, XX(4), 597-614. doi: 10.1093/ojls/20.4.597 
Breyer, S. G., Stewart, R. B., Sunstein, C. R. y Vermeule, A. (2006). Administrative Law and Regulatory Policy: Problems, Text, and Cases (6th ed.). New York: Aspen Publishers.

Brown, C., y Scott, C. (2011). Regulation, Public Law, and Better Regulation. European Public Law, 17(3), 467-484. Obtenido de Dialnet: http://dialnet.unirioja.es/servlet/ articulo?codigo $=3738103$

Comisión Europea. (2010a). COM (2010) 543 Final. Comunicación de la Comisión al Parlamento Europeo, al Consejo, al Comité Económico y Social Europeo y al Comité de las Regiones. Normativa inteligente en la Unión Europea. Bruselas.

Comisión Europea. (2010b). COM (2010) 2020. Europa 2020: Una estrategia para un crecimiento inteligente, sostenible e integrador. Bruselas.

Departamento Nacional de Planeación. (2013). Boletín de Prensa. Bogotá, octubre 25. Obtenido de DNP: https://pwh.dnp.gov.co/LinkClick.aspx?fileticket=QhA2rBviGL0\%3D\&tab id $=1658$

Dubash, N. K. y Morgan, B. (2012). Understanding the rise of the regulatory state of the South. Regulation \& Governance, 6(3), 261281. doi:10.1111/j.1748-5991.2012.01146.x

Friedman, L. M. (1994). Is There a Modern Legal Culture? Ratio Juris, 7(2), 117-131. Obtenido de Wiley Online Library: http://onlinelibrary. wiley.com/doi/10.1111/j.1467-9337.1994. tb00172.x/abstract

Fuller, L. L. (1969). The Morality of Law: Revised Edition. New Haven: Yale University Press.

García Villegas, M. (2002). Notas preliminares para la caracterización del derecho en América Latina. El Otro Derecho, (26-27), 13-48. Obtenido de ilsa.org: http://ilsa.org.co:81/ biblioteca/dwnlds/od/elotrdr026-27/elotrdr026-27-01.pdf.

Gargarella, R. (1998). Full representation, deliberation and impartiality. In J. Elster (Ed.), Deliberative democracy (pp. 260-280). Cambridge: Cambridge University Press.

Jordana, J. y Levi-faur, D. (2004). The politics of regulation in the age of governance. En J. Jordana y D. Levi-Faur (Ed.), The Politics Of Regulation: Institutions and Regulatory Reforms for the Age of Governance (pp. 1-28). Cheltenham: Edward Elgar Publishing.

Jordana, J. y Sancho, D. (2004). Regulatory designs, institutional constellations and the study of the regulatory state. The politics of regulation in the age of governance. En J. Jordana y D. Levi-Faur (Ed.), The Politics of Regulation: Institutions and Regulatory Reforms for the Age of Governance (pp. 296-319). Cheltenham: Edward Elgar Publishing.

Latorre, I. (2013). Tendencias internacionales para la mejora de la producción normativa. (Manuscrito sin publicar). 
Lodge, M. (2004). Accountability and transparency in regulation: critiques, doctrines and instruments. En J. Jordana and D. Levi-Faur (Ed.), The Politics of Regulation: Institutions and Regulatory Reforms for the Age of Governance (pp. 124-144). Cheltenham: Edward Elgar Publishing.

López-Murcia, J. D. (2013). Regulatory Agencies and Courts in the South: The Overlaps in Colombian Water Regulation. Journal of Politics in Latin America, 5(2), 105-132. Obtenido de Journal of Politics in Latin America: http://journals.sub.uni-hamburg.de/giga/ jpla/article/view/664

Luban, D. J. (2002). The Publicity of Law and the Regulatory State. Journal of Political Philosophy, 10(3), 296-316. doi: 10.1111/14679760.00154

Majone, G. (1997). From the Positive to the Regulatory State: Causes and Consequences of Changes in de Mode of Governance. Journal of Public Policy, 17(2), 139-167. doi: 10.1017/ S0143814X00003524

Malyzhev, N. (2006). Regulatory Policy: OECD Experience and Evidence. Oxford Review of Economic Policy, 22(2), 274-299. 0.1093/ oxrep/grj017

Mandelkern Group on Better Regulation. (13 de noviembre de 2001). Final Report. (p. 84).

Martí, J. L. (2005). The Sources of Legitimacy of Political Decisions: Between Procedure and Substance. En L. J. Wintgens (Ed.), The Theory and Practice of Legislation: Essays in Legisprudence (pp. 259-284). London: Ashgate.

Martí, J. L. (2006). La república deliberativa: una teoría de la democracia. Madrid, Barcelona: Marcial Pons.

Meuwese, A. C. (2008). Impact assessment in EU lawmaking. The Hague: Kluwer Law International.

Meuwese, A. y Popelier, P. (2011). Legal Implications of Better Regulation: A Special Issue. European Public Law, 17(3), 455-466. Obtenido de Dialnet: http://dialnet.unirioja. es/servlet/articulo?codigo $=3738101$

Morelo, G., Báez, C. y Garibello, A. (20 de julio de 2014). Balance del Congreso en cuatrienio: 8 de cada 10 leyes se archivaron. Obtenido de El Tiempo: http://www.eltiempo.com/ politica/congreso/balance-del-congreso-entre-

Organización para la Cooperación y el Desarrollo Económicos. (9 de marzo de 1995). C(95)21/ FINAL. Recommendation of the Council on Improving the Quality of Government Regulation. OECD Publishing.

Organización para la Cooperación y el DesarroIlo Económicos. (2012). Recomendación del Consejo sobre Política y Gobernanza Regulatoria. OECD Publishing.

Organización para la Cooperación y el DesarroIlo Económicos. (2013a). Regulatory Policy in Colombia: Going beyond Administrative Simplification. OECD Publishing. 
Organización para la Cooperación y el Desarrollo Económicos. (2013b). Revisiones de la OCDE sobre Reforma Regulatoria. OECD Publishing.

Pierce, R. J. J. (1995). Seven Ways to Deossify Agency Rulemaking. Administrative Law Review, 47(1), 59 - 95.

Obtenido de heinonline: http://heinonline.org/HOL/Page?handle=hein.journals / admin $47 \&$ id $=69 \&$ div $=\&$ collecti on=journals

Posner, E. A. y Vermeule, A. (2010). The Executive Unbound: After the Madisonian Republic. New York: Oxford University Press.

Prosser, T. (2010). The Regulatory enterprise: government, regulation, and legitimacy. New York: Oxford University Press.

Radaelli, C. M. (2007). Towards better research on better regulation. Paper delivered to the Advanced Colloquium on Better Regulation, Centre for regulatory Governance, University of Exeter, 25- 26 January. Obtenido de University of Exeter: http://socialsciences. exeter.ac.uk/politics/research/readingroom/ radaelliTowardsBett erResearch.doc.

Radaelli, C. M. y De Francesco, F. (2007). Regulatory quality in Europe: Concepts, measures and policy processes. New York: Manchester University Press.

Radaelli, C. M., De Francesco, F. y Troeger, V. E. (2008). The Implementation of Regulatory Impact Assessment in Europe. Paper delivered to the ENBR workshop. Exeter: University of Exeter, 27 and 28 March.
Rey Salamanca, F. (2014). Teoría de la representación política en el derecho público. Bogotá: Grupo Editorial Ibáñez, Pontificia Universidad Javeriana.

Scott, C. (2004). Regulation in the age of governance: the rise of the post-regulatory state. En J. Jordana y D. Levi-Faur (Ed.), The Politics of Regulation: Institutions and Regulatory Reforms for the Age of Governance (pp. 145174). Cheltenham: Edward Elgar Publishing.

Scott, C. (2010). Regulatory Governance and the Challenge of Constitutionalism. En D. Oliver, T. Prosser y R. Rawlings (Eds.), The Regulatory State. Constitutional Implications (pp. 1533). New York: Oxford University Press.

Seidenfeld, M. (1997). Demystifying deossification: Rethinking recent proposals to modify judicial review of notice and comment rulemaking. Texas Law Review, 75(3), 483-524. Obtenido de Social Science Research Network: http://papers.ssrn.com/sol3/papers. cfm?abstract_id=1106309

Shapiro, S., Fisher, E. y Wagner, W. (2012). The Enlightenment of Administrative Law: Looking Inside the Agency for Legitimacy. Forest Law Review, 47(3), 463-502. Obtenido de The Wake Forest Law Review: http://wakeforestlawreview.com/wp-content/ uploads/2012/10/w03_SFW.pdf.

Shearing, C. y Wood, J. (2003). Nodal Governance, Democracy, and the New 'Denizens'. Journal of Law and Society, 30(3), 400-419. doi:10.1111/1467-6478.00263 
Sunstein, C. R. (1990). After the rights revolution: Reconceiving the regulatory state. Cambridge: Harvard University Press.

Sunstein, C. R. (1996). The Cost-Benefit State. Chicago Working Papers in Law and Economics, 39. Obtenido de University of Chicago Law School: http://chicagounbound.uchicago.edu/cgi/viewcontent. cgi?article=1497\&context=law_and_economics

Sunstein, C. R. (2014). Valuing Life: Humanizing the Regulatory State. Chicago: University of Chicago Press.

Sunstein, C. R. y Lebrón, J. M. (2006). Riesgo y razón: seguridad, ley y medioambiente. Buenos Aires: Katz.

Tala, J. (Octubre de 2010). Better Regulation Through Programs and Quality Standards-Are New Perspectives Needed? Legisprudence, 4(2), 193-212. Obtenido de ingentaconnect: http://www.ingentaconnect.com/content/ hart/legis/2010/00000004/00000002/ art00006

Tamanaha, Brian Z. (2006). Law as a means to an end: threat to the rule of law. Cambridge: Cambridge University Press.

Toner, H. (2006). Impact assessments and fundamental rights protection in EU Law. European Law Review, 3(1), 316-341. Obtenido de Dialnet: http://dialnet.unirioja.es/servlet/ articulo?codigo $=2033402$

Urueña, R. (2012). The rise of the constitutional regulatory state in Colombia: The case of water governance. Regulation \& Governance, 6(3), 282-299. doi: 10.1111/j.17485991.2012.01139.x

Verkuil, P.R. (1995). Comment: Rulemakingossification - A modest proposal. Administrative Law Review,47,453-459. Obtenido deHeinOnline:http:// www.heinonline.org/HOL/Page?handle=hein. journals / admin $47 \&$ id $=463 \&$ collect ion=journals\&index=journals/admin

Vibert, F. (2011). Democracy and Dissent: The Challenge of international Rule Making. Cheltenham: Edward Elgar Publishing.

Vibert, F. (2014). The New Regulatory Space. Reframing Democratic Governance. Cheltenham, U.K.: Edward Elgar Publishing.

Voermans, W. (2009). Concern about the Quality of EU Legislation: What Kind of Problem, by What Kind of Standards? Erasmus Law Rev, 2(1), 59-95. Obtenido de erasmuslawreview: http://www.erasmuslawreview.nl/files/ ELR_issue6_004.pdf

Wintgens, L. J. (Marzo de 2006). Legisprudence as a New Theory of Legislation. Ratio Juris, 19(1), 1-25. doi: 10.1111/j.14679337.2006.00315.x 